\title{
An Intelligent Fuzzy-based Cluster Head Selection System for Wireless Sensor Networks and Its Performance Evaluation
}

\author{
Hironori Ando*, Leonard Barolli ${ }^{\dagger}$, Arjan Durresi ${ }^{\ddagger}$, Fatos Xhafa $^{\S}$, and Akio Koyama $₫$ \\ * Graduate School of Engineering \\ Fukuoka Institute of Technology (FIT) \\ 3-30-1 Wajiro-Higashi, Higashi-Ku, Fukuoka 811-0295, Japan \\ E-mail: mgm10001@bene.fit.ac.jp \\ ${ }^{\dagger}$ Department of Information and Communication Engineering \\ Fukuoka Institute of Technology (FIT) \\ 3-30-1 Wajiro-Higashi, Higashi-Ku, Fukuoka 811-0295, Japan \\ E-mail: barolli@fit.ac.jp \\ $\ddagger_{\text {Department of Computer and Information Science }}$ \\ Indiana University Purdue University at Indianapolis (IUPUI) \\ 723 W. Michigan Street SL 280, Indianapolis, IN 46202, USA \\ E-mail:durresi@cs.iupui.edu \\ $\S$ Department of Languages and Informatics Systems \\ Technical University of Catalonia \\ Jordi Girona 1-3, 08034 Barcelona, Spain \\ E-mail:fatos@lsi.upc.edu \\ IDepartment of Informatics, Yamagata University \\ 4-3-16 Jonan, Yonezawa 992-8510, Yamagata, Japan \\ E-mail: akoyama@eie.yz.yamagata-u.ac.jp
}

\begin{abstract}
Sensor networks supported by recent technological advances in low power wireless communications along with silicon integration of various functionalities are emerging as a critically important computer class that enable novel and low cost applications. There are many fundamental problems that sensor networks research will have to address in order to ensure a reasonable degree of cost and system quality. Cluster formation and cluster head selection are important problems in sensor network applications and can drastically affect the network's communication energy dissipation. However, selecting of the cluster head is not easy in different environments which may have different characteristics. In our previous work, in order to deal with this problem, we proposed a power reduction algorithm for sensor networks based on fuzzy logic and number of neighbor nodes. We call this algorithm F3N. In this paper, we implement a simulation system for clustering algorithms in sensor networks. We evaluate LEACH and F3N by some simulation results. Presently, we have implemented LEACH algorithm in NS-2. However, F3N is implemented in MATLAB. We are working to implement also F3N system in NS-2 in order to compare their performance.
\end{abstract}

Keywords-Sensor Networks; Clustering Algorithms; Fuzzy Logic; NS-2.

\section{INTRODUCTION}

Recent developments in technologies such as wireless communication and microelectronics have enabled Wireless Sensor Network (WSN) applications to be deployed for many applications such as battlefield surveillance and environment monitoring. An important aspect of such networks is that the nodes are unattended, resource-constrained, their energy cannot be replenished and network topology is unknown. The resource-constrained limitations make it essential for these sensor nodes to conserve energy to increase life-time of the sensor network [1], [2], [3], [4].

Recently, there are lot of research efforts towards the optimization of standard communication paradigms for such networks. In fact, the traditional Wireless Network (WN) design has never paid attention to constraints such as the limited or scarce energy of nodes and their computational power. Also, in WSN paths can change over time, because of time-varying characteristics of links, local contention level and nodes reliability. These problems are important 
especially in a multi-hop scenario, where nodes accomplish also at the routing of other nodes' packets [4].

There are many fundamental problems that sensor networks research will have to address in order to ensure a reasonable degree of cost and system quality. Some of these problems include sensor node clustering, Cluster Head $(\mathrm{CH})$ selection and energy dissipation. There are many research works that deal with these challenges [5], [6], [7], [8], [9], [10], [11], [12], [13].

The cluster based algorithms could be used for partitioning the sensor nodes into subgroups for task subdivision or energy management. Cluster formation is one of most important problems in sensor network applications and can drastically affect the network's communication energy dissipation. Clustering is performed by assigning each sensor node to a specific $\mathrm{CH}$. All communication to (from) each sensor node is carried out through its corresponding $\mathrm{CH}$ node. Obviously one would like to have each sensor to communicate with the closest $\mathrm{CH}$ node to conserve its energy, however $\mathrm{CH}$ nodes can usually handle a specific number of communication channels. Therefore, there is a maximum number of sensors that each $\mathrm{CH}$ node can handle. This does not allow each sensor to communicate to its closest $\mathrm{CH}$ node, because the $\mathrm{CH}$ node might have already reached its service capacity. $\mathrm{CHs}$ can fuse data from sensors to minimize the amount of data to be sent to the sink. When network size increases, clusters can also be organized hierarchically.

In the conventional cluster architecture, clusters are formed statically at the time of network deployment. The attributes of each cluster, such as the size of a cluster, the area it covers, and the members it possesses, are static. In spite of its simplicity, the static cluster architecture suffers from several drawbacks. The fixed membership is not robust from the perspective of fault tolerance. If a $\mathrm{CH}$ dies of power depletion, all the sensors in the cluster render useless. Also, fixed membership prevents sensor nodes in different clusters from sharing information and collaborating on data processing. Dynamic cluster architectures, on the other hand, offer several desirable features. Formation of a cluster is triggered by certain events of interest.

When a sensor with sufficient battery and computational power detects (with a high Signal-to-Noise Ratio: SNR) signals of interest, it volunteers to act as a $\mathrm{CH}$. This is a simple method, because no explicit leader $(\mathrm{CH})$ election is required and, hence, no excessive message exchanges are incurred. However, selecting of the $\mathrm{CH}$ in this way is not easy in different environments which may have different characteristics such as error rate, SNR, throughput and so on.

The heuristic approaches based on Fuzzy Logic (FL) and Genetic Algorithms (GA) can prove to be efficient for traffic control in wireless networks [14], [15].

In our previous work [16], we proposed a fuzzy-based cluster selection method for wireless sensor networks, which uses 3 parameters for $\mathrm{CH}$ selection: Distance of Cluster Centroid, Remaining Battery Power of Sensor and Network Traffic. The performance of our method was better than a previous method [17]. But, we found that for $\mathrm{CH}$ selection the number of neighbor nodes is very important. For this reason, we propose and implement a new $\mathrm{CH}$ system using FL and number of neighbor nodes called F3N [18], [19].

In this work, we implement a simulation system for clustering algorithms in sensor networks. We evaluate LEACH and F3N by some simulation results. Presently, we have implemented LEACH algorithm in NS-2. However, F3N is implemented in MATLAB. We are working to implement also F3N system in NS-2 in order to compare their performance.

The paper is organized as follows. In Section 2, we discuss the related work. In Section 3, we introduce the proposed system. In Section 4, we present the simulation results. Conclusions are given in Section 5.

\section{RELATED WORK}

In this section, we review related work in clustering algorithms. Several clustering methods such as weighted clustering [5], hierarchal clustering [6] and dynamic clustering [7] algorithms have been proposed to organize nodes as a cluster. Most algorithms select leaders based on certain weights or iteratively optimize a cost function or use heuristic to generate minimum number of clusters. The Distributed Clustering Algorithm (DCA) [8] assumes quasistationary nodes with real-valued weights. The Weighted Clustering Algorithm [5] elects a node based on the number of neighbors, transmission power and so on. The Max-Min d-Clustering Algorithm [9] generates d-hop clusters with a run time of $\mathrm{O}(\mathrm{d})$ rounds. This algorithm does not minimize the communicating complexity of sending information to the information center.

The hierarchical clustering scheme [6] uses spanning treebased approach to produce cluster with certain properties. However, energy efficiency is not addressed in this work. In [10], the authors have proposed an emergent algorithm that iteratively tries to achieve high packing efficiency, however negotiation among nodes to be $\mathrm{CH}$ and join cluster based on degree and proximity leads to high amount of communication overhead, thus wastage energy.

LEACH uses two-layered architecture for data dissemination. In this scheme, sensors periodically elect themselves as $\mathrm{CHs}$ with some probability and broadcast an invitation message for nearby nodes to join the cluster. The nodes that do not intend to be $\mathrm{CHs}$ join the cluster based on the proximity of $\mathrm{CH}$, thus minimizing the communicating cost. However, LEACH and PEGASIS [13] require a priori knowledge of the network topology.

In [17], the authors propose a self-reconfiguring protocol for Wireless Personal Area Networks (WPAN) using an 


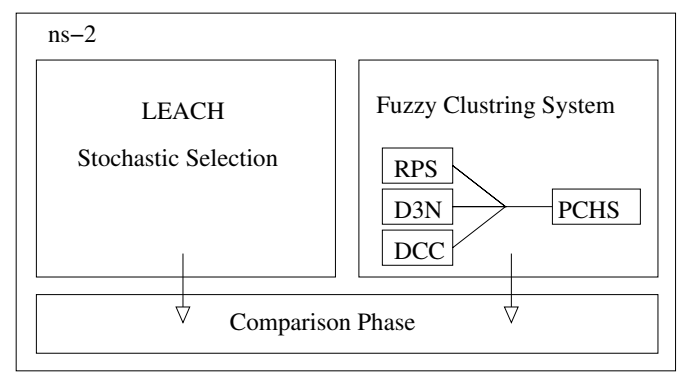

Figure 1. Proposed simulation system.

unsupervised clustering method. A fuzzy logic system is used to select the master/controller for each cluster. In our previous work [16], we had shown by simulation results that the selection surface of our system was better than the system in [17]. But, we found that for $\mathrm{CH}$ selection the number of neighbor nodes is very important. For this reason, we proposed and implemented a new $\mathrm{CH}$ system using FL and number of neighbor nodes called F3N [18], [19].

\section{Proposed System}

\section{A. Structure of Proposed Simulation System}

The structure of the proposed system is shown in Fig. 1. The LEACH protocol has 2 phases: advertisement phase and cluster set-up phase. In the advertisement phase of $\mathrm{LEACH}$, the $\mathrm{CH}$ is selected for each round based on a defined expression. While, in F3N system, the $\mathrm{CH}$ is selected based on FL. The cluster set-up phase is the same for both systems.

Presently, we have implemented LEACH protocol in NS-2 and are working now to implement also F3N system in NS-2 in order to make a fair comparison between both systems.

\section{B. LEACH Algorithm}

The operation of LEACH is broken up into rounds, where each round begins with a set-up phase, when the clusters are organized, followed by a steady-state phase, when data transfers to the base station occur. In order to minimize overhead, the steady-state phase is long compared to the set-up phase.

1) Advertisement Phase: Initially, when clusters are being created, each node decides whether or not to become a $\mathrm{CH}$ for the current round. This decision is based on the suggested percentage of $\mathrm{CHs}$ for the network (determined a priori) and the number of times the node has been a $\mathrm{CH}$ so far. This decision is made by the node $n$ choosing a random number between 0 and 1 . If the number is less than a threshold $T(n)$, the node becomes a $\mathrm{CH}$ for the current round. The threshold is set as:

$$
T(i)= \begin{cases}\frac{P}{1-P \cdot\left(r \bmod \frac{1}{P}\right)} & \text { if } i \in G \\ 0 & \text { otherwise }\end{cases}
$$

where $P=$ the desired percentage of $\mathrm{CHs}$ (e.g., $P=0: 05$ ), $r=$ the current round, and $G$ is the set of nodes that have not been $\mathrm{CHs}$ in the last $\frac{1}{P}$ rounds. Using this threshold, each node will be a $\mathrm{CH}$ at some point within $\frac{1}{P}$ rounds. During round $0(r=0)$, each node has a probability $P$ of becoming a $\mathrm{CH}$. The nodes that are $\mathrm{CHs}$ in round 0 cannot be CHs for the next $\frac{1}{P}$ rounds. Thus, the probability that the remaining nodes are $\mathrm{CHs}$ must be increased, since there are fewer nodes that are eligible to become CHs. After $\frac{1}{P}-1$ rounds, $T=1$ for any nodes that have not yet been $\mathrm{CHs}$, and after $\frac{1}{P}$ rounds, all nodes are once again eligible to become CHs.

Each node that has elected itself a $\mathrm{CH}$ for the current round broadcasts an advertisement message to the rest of the nodes. For this "CH-advertisement" phase, the CHs use a CSMA MAC protocol, and all $\mathrm{CHs}$ transmit their advertisement using the same transmit energy. The non$\mathrm{CH}$ nodes must keep their receivers on during this phase of set-up to hear the advertisements of all the $\mathrm{CH}$ nodes. After this phase is complete, each non- $\mathrm{CH}$ node decides the cluster to which it will belong for this round. This decision is based on the received signal strength of the advertisement. Assuming symmetric propagation channels, the $\mathrm{CH}$ advertisement heard with the largest signal strength is the $\mathrm{CH}$ to whom the minimum amount of transmitted energy is needed for communication. In the case of ties, a random $\mathrm{CH}$ is chosen.

2) Cluster Set-Up Phase: After each node has decided to which cluster it belongs, it must inform the $\mathrm{CH}$ node that it will be a member of the cluster. Each node transmits this information back to the $\mathrm{CH}$ again using a CSMA MAC protocol. During this phase, all $\mathrm{CH}$ nodes must keep their receivers on.

3) Schedule Creation: The $\mathrm{CH}$ node receives all the messages for nodes that would like to be included in the cluster. Based on the number of nodes in the cluster, the $\mathrm{CH}$ node creates a TDMA schedule telling each node when it can transmit. This schedule is broadcast back to the nodes in the cluster.

4) Data Transmission: Once the clusters are created and the TDMA schedule is fixed, data transmission can begin. Assuming nodes always have data to send, they send it during their allocated transmission time to the $\mathrm{CH}$. This transmission uses a minimal amount of energy (chosen based on the received strength of the $\mathrm{CH}$ advertisement). The radio of each non- $\mathrm{CH}$ node can be turned off until the node's allocated transmission time, thus minimizing energy dissipation in these nodes. The $\mathrm{CH}$ node must keep its receiver on to receive all the data from the nodes in the cluster. When all the data has been received, the $\mathrm{CH}$ node performs signal processing functions to compress the data into a single signal. For example, if the data are audio or seismic signals, the $\mathrm{CH}$ node can beam form the individual signals to generate a composite signal. This composite signal 


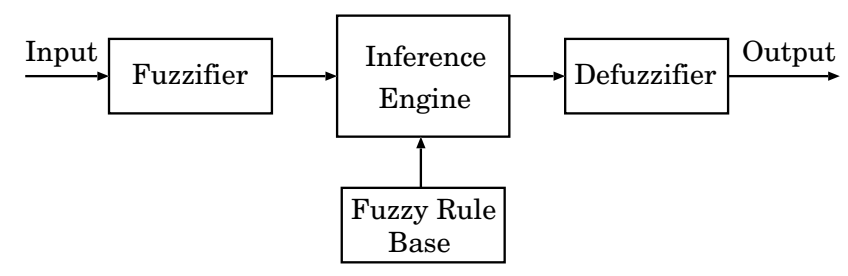

Figure 2. FLC structure.
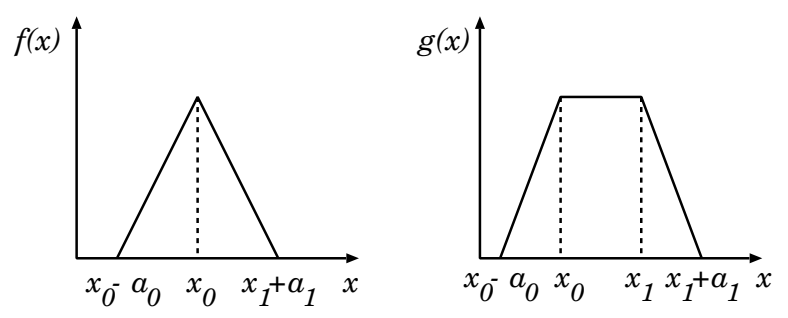

Figure 3. Triangular and trapezoidal membership functions.

is sent to the base station. Since the base station is far away, this is a high-energy transmission. This is the steadystate operation of LEACH. After a certain time, which is determined a priori, the next round begins with each node determining if it should be a $\mathrm{CH}$ for this round.

\section{F3N System}

Here we present F3N system. The Fuzzy Logic Controller (FLC) basic elements are shown in Fig. 2. They are the fuzzifier, inference engine, Fuzzy Rule Base (FRB) and defuzzifier.

As shown in Fig. 3, as membership functions we use triangular and trapezoidal membership functions because they are suitable for real-time operation [20].

They are shown in Fig. 3 and are given as:

$$
\begin{gathered}
f\left(x ; x_{0}, a_{0}, a_{1}\right)= \begin{cases}\frac{x-x_{0}}{a_{0}}+1 & \text { for } x_{0}-a_{0}<x \leq x_{0} \\
\frac{x_{0}-x}{a_{1}}+1 & \text { for } x_{0}<x \leq x_{0}+a_{1} \\
0 & \text { otherwise }\end{cases} \\
g\left(x ; x_{0}, x_{1}, a_{0}, a_{1}\right)= \begin{cases}\frac{x-x_{0}}{a_{0}}+1 & \text { for } x_{0}-a_{0}<x \leq x_{0} \\
1 & \text { for } x_{0}<x \leq x_{1} \\
\frac{x_{1}-x}{a_{1}}+1 & \text { for } x_{1}<x \leq x_{1}+a_{1} \\
0 & \text { otherwise }\end{cases}
\end{gathered}
$$

where, $x_{0}$ in $f(x)$ is the center of triangular function; $x_{0}\left(x_{1}\right)$ in $g(x)$ is the left (right) edge of trapezoidal function; and $a_{0}\left(a_{1}\right)$ is the left (right) width of the triangular or trapezoidal function.

In our previous system as the input parameters for FLC we used: Distance of Cluster Centroid, Remaining Battery Power of Sensor and Network Traffic. However, we found that the number of the neighbor nodes is very important for the selection of the $\mathrm{CH}$. To explain this effect let us consider a small network model with 14 nodes as shown in Fig. 4. In this figure, the node number 1 has 6 neighbor nodes, for this reason is selected as a $\mathrm{CH}$. After that, 2 other sets of nodes

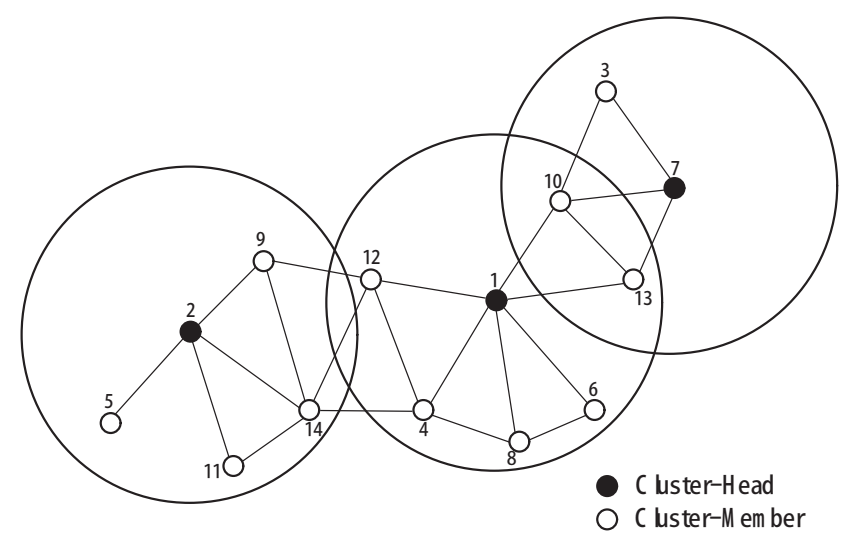

Figure 4. $\mathrm{CH}$ selection process.

remain, but node 2 has more neighbor nodes that nodes 3 and 7, thus node 2 is selected as CH. Finally, if we see 2 other remained nodes (node 3 and node 7), they have the same neighbor nodes that are not included in other clusters. In this case, the node with higher ID number is selected as $\mathrm{CH}$. For this reason, node 7 is selected as $\mathrm{CH}$ and the procedure of $\mathrm{CH}$ selection is finished.

By considering the number of neighbor nodes as a fuzzy input parameter, we propose 3 input linguistic parameters for our system:

- Remaining Battery Power of Sensor (RPS);

- Degree of Number of Neighbor Nodes (D3N);

- Distance from Cluster Centroid (DCC).

The term sets for each input linguistic parameter are defined respectively as:

$$
\begin{aligned}
T(R P S) & =\{\operatorname{Low}(\text { Lo }), \text { Middle }(M i), \operatorname{High}(H g)\} ; \\
T(D 3 N) & =\{\text { Few }(F w), \text { Medium }(M e), \operatorname{Many}(M n)\} ; \\
T(D C C) & =\{\text { Near }(N r), \text { Moderate }(M o), \operatorname{Far}(F r)\} .
\end{aligned}
$$

The membership functions for input parameters of FLC are defined as:

$$
\begin{aligned}
\mu_{L o}(R P S) & =g\left(R P S ; L o_{0}, L o_{1}, L o_{w 0}, L o_{w 1}\right) \\
\mu_{M i}(R P S) & =f\left(R P S ; M i_{0}, M i_{w 0}, M i_{w 1}\right) ; \\
\mu_{H g}(R P S) & =g\left(R P S ; H g_{0}, H g_{1}, H g_{w 0}, H g_{w 1}\right) ; \\
\mu_{F w}(D 3 N) & =g\left(D 3 N ; F w_{0}, F w_{1}, F w_{w 0}, F w_{w 1}\right) ; \\
\mu_{M e}(D 3 N) & =f\left(D 3 N ; M e_{0}, M e_{w 0}, M e_{w 1}\right) ; \\
\mu_{M n}(D 3 N) & =g\left(D 3 N ; M n_{0}, M n_{1}, M n_{w 0}, M n_{w 1}\right) ; \\
\mu_{N r}(D C C) & =g\left(D C C ; N r_{0}, N r_{1}, N r_{w 0}, N r_{w 1}\right) ; \\
\mu_{M o}(D C C) & =f\left(D C C ; M o_{0}, M o_{w 0}, M o_{w 1}\right) ; \\
\mu_{F r}(D C C) & =g\left(D C C ; F r_{0}, F r_{1}, F r_{w 0}, F r_{w 1}\right)
\end{aligned}
$$

The small letters $w 0$ and $w 1$ mean left width and right width, respectively.

The output linguistic parameter is the Possibility of $\mathrm{CH}$ Selection $(P C H S)$. We define the term set of $P C H S$ as: $\{$ Very Weak $(V W)$, Weak $(W)$, Little Weak $(L W)$, 
Table I

PARAMETERS AND THEIR TERM SETS.

\begin{tabular}{|l|l|}
\hline Parameters & Term Sets \\
\hline $\begin{array}{l}\text { Remaining Battery } \\
\text { Power of Sensor (RPS) } \\
\begin{array}{l}\text { Degree of Number of } \\
\text { Neighbor Nodes (D3N) }\end{array}\end{array}$ & Low, Middle, High \\
$\begin{array}{l}\text { Distance from Cluster } \\
\text { Centroid (DCC) }\end{array}$ & Light, Moderate, Heavy \\
\hline $\begin{array}{l}\text { Probability (Possibility) } \\
\text { of CH Selection (PCHS) }\end{array}$ & $\begin{array}{l}\text { Very Weak, Weak, Little } \\
\text { Week, Medium, Little Strong, } \\
\text { Strong, Very Strong }\end{array}$ \\
\hline
\end{tabular}

Medium (MD), Little Strong $(L S)$, Strong $(S)$, Very Strong $(V S)\}$.

The membership functions for the output parameter $\mathrm{PCHS}$ are defined as:

$$
\begin{aligned}
\mu_{V W}(P C H S) & =g\left(P C H S ; V W_{0}, V W_{1}, V W_{w 0}, V W_{w 1}\right) ; \\
\mu_{W}(P C H S) & =f\left(P C H S ; W_{0}, W_{w 0}, W_{w 1}\right) ; \\
\mu_{L W}(P C H S) & =f\left(P C H S ; L W_{0}, L W_{w 0}, L W_{w 1}\right) ; \\
\mu_{M D}(P C H S) & =f\left(P C H S ; M D_{0}, M D_{w 0}, M D_{w 1}\right) ; \\
\mu_{L S}(P C H S) & =f\left(P C H S ; L S_{0}, L S_{w 0}, L S_{w 1}\right) ; \\
\mu_{S}(P C H S) & =f\left(P C H S ; S_{0}, S_{w 0}, S_{w 1}\right) ; \\
\mu_{V S}(P C H S) & =g\left(P C H S ; V S_{0}, V S_{1}, V S_{w 0}, V S_{w 1}\right) .
\end{aligned}
$$

The linguistic parameters and their term sets of proposed system are shown in Table 1. The fuzzy membership functions for input parameters are shown in Fig. 5.

The FRB is shown in Table 2 and forms a fuzzy set of dimensions $|T(R P S)| \times|T(D 3 N)| \times|T(D C C)|$, where $|T(x)|$ is the number of terms on $T(x)$. The FRB has 27 rules. The control rules have the form: IF "conditions" THEN "control action".

\section{Simulation Results}

\section{A. Simulation Results for LEACH}

In Fig. 6, we show the simulation results of LEACH protocol when it uses the energy only for sensing. We carried out the simulations for 20 nodes and the simulations were run 50 times. As can be seen by this figure all the nodes die almost at the same time for about 615 seconds.

In Fig. 7, we show the simulation results for LEACH protocol when the nodes communicate with each other. We see that the number of nodes which remained alive decreases with increase of simulation time.

\section{B. Simulation Results for F3N}

In this section, we present the simulation results for $\mathrm{F} 3 \mathrm{~N}$ system. In our system, we decided the number of term sets by carrying out many simulations. These simulation results were carried out in MATLAB.

We show the performance of F3N in Fig. 8, Fig. 9 and Fig. 10. In Fig. 8 is shown the relation between the probability of a sensor to be selected as a CH versus the DCC and RPS. We clearly distinguish 3 zones. When, the RPS is less than

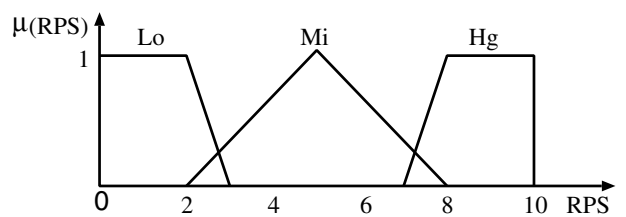

\begin{tabular}{|c|c|c|c|c|}
\hline Rule & $\overline{\mathrm{RPS}}$ & $\overline{\mathrm{D} 3 \mathrm{~N}}$ & $\overline{\mathrm{DCC}}$ & PCHS \\
\hline 1 & Lo & Fw & $\mathrm{Nr}$ & VW \\
\hline 2 & Lo & $\mathrm{FW}_{\mathrm{W}}$ & Мо & W \\
\hline 3 & Lo & Fw & $\mathrm{Fr}$ & W \\
\hline 4 & Lo & $\mathrm{Me}$ & $\mathrm{Nr}$ & W \\
\hline 5 & Lo & $\mathrm{Me}$ & Mo & W \\
\hline 6 & Lo & $\mathrm{Me}$ & $\mathrm{Fr}$ & W \\
\hline 7 & Lo & $\mathrm{Mn}$ & $\mathrm{Nr}$ & VW \\
\hline 8 & Lo & $\mathrm{Mn}$ & Mo & VW \\
\hline 9 & Lo & $\mathrm{Mn}$ & $\mathrm{Fr}$ & VW \\
\hline 10 & $\mathrm{Mi}$ & Fw & $\mathrm{Nr}$ & W \\
\hline 11 & $\mathrm{Mi}$ & Fw & Mo & LW \\
\hline 12 & Mi & Fw & $\mathrm{Fr}$ & MD \\
\hline 13 & $\mathrm{Mi}$ & $\mathrm{Me}$ & $\mathrm{Nr}$ & LW \\
\hline 14 & $\mathrm{Mi}$ & $\mathrm{Me}$ & Mo & MD \\
\hline 15 & $\mathrm{Mi}$ & $\mathrm{Me}$ & $\mathrm{Fr}$ & LS \\
\hline 16 & Mi & Mn & $\mathrm{Nr}$ & MD \\
\hline 17 & $\mathrm{Mi}$ & $\mathrm{Mn}$ & Mo & LS \\
\hline 18 & $\mathrm{Mi}$ & $\mathrm{Mn}$ & $\mathrm{Fr}$ & S \\
\hline 19 & $\mathrm{Hg}$ & $\mathrm{Fw}$ & $\mathrm{Nr}$ & LW \\
\hline 20 & $\mathrm{Hg}$ & FW & Mo & MD \\
\hline 21 & $\mathrm{Hg}$ & Fw & $\mathrm{Fr}$ & LS \\
\hline 22 & $\mathrm{Hg}$ & $\mathrm{Me}$ & $\mathrm{Nr}$ & MD \\
\hline 23 & $\mathrm{Hg}$ & $\mathrm{Me}$ & Mo & LS \\
\hline 24 & $\mathrm{Hg}$ & $\mathrm{Me}$ & $\mathrm{Fr}$ & S \\
\hline 25 & $\mathrm{Hg}$ & $\mathrm{Mn}$ & $\mathrm{Nr}$ & LS \\
\hline 26 & $\mathrm{Hg}$ & $\mathrm{Mn}$ & Mo & S \\
\hline 27 & $\mathrm{Hg}$ & $\mathrm{Mn}$ & $\mathrm{Fr}$ & VS \\
\hline
\end{tabular}
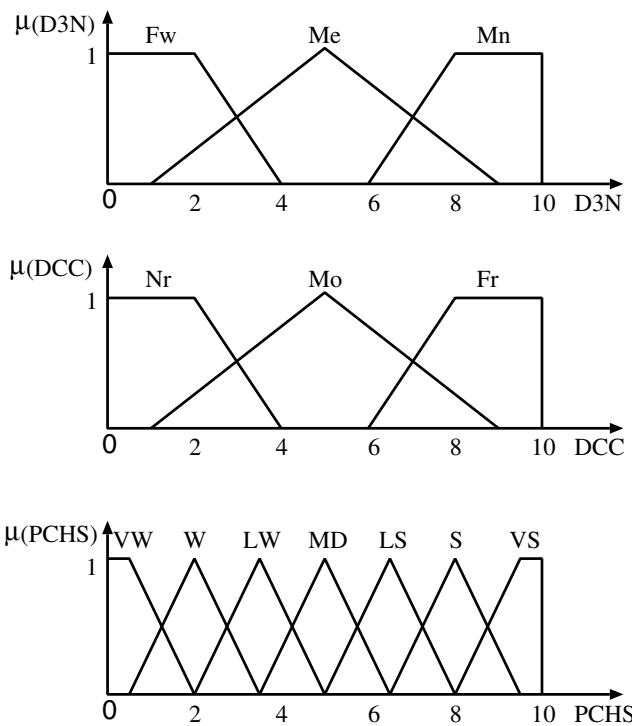

Figure 5. Membership functions. Table II FRB.

2 units the probability of a node to be selected as $\mathrm{CH}$ is very small. A middle zone (more than 2 units but less than 8 units), where the $\mathrm{CH}$ selection possibility increases slowly 


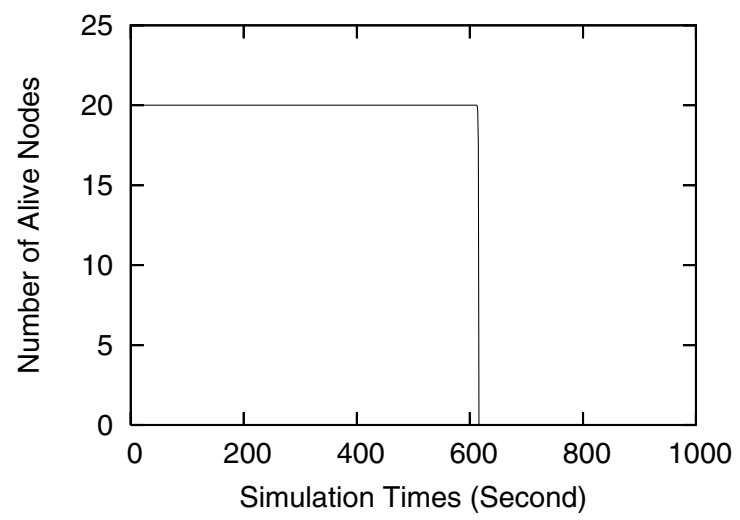

Figure 6. LEACH simulation results only for sensing.

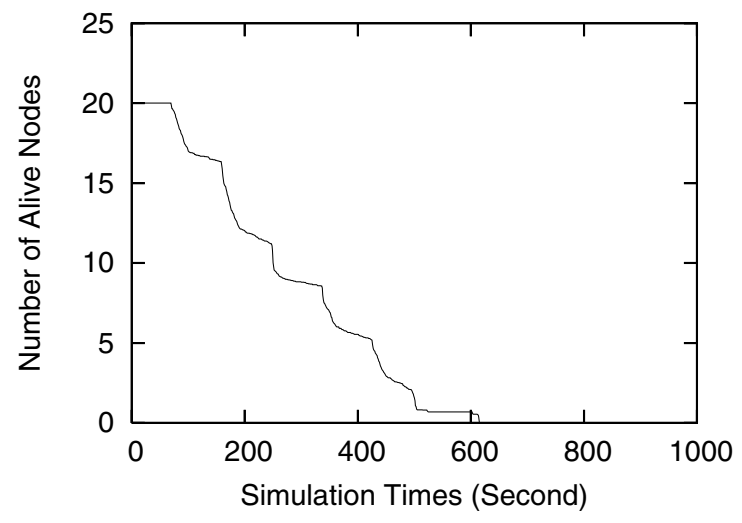

Figure 7. LEACH simulation results.

with increase of the RPS and decrease of DCC. A third zone (more than 8 units), where the possibility of a node to be $\mathrm{CH}$ is high. In this way, the proposed system can control better the RPS, which results in the increase of the network lifetime.

In Fig. 9 is shown the relation between the probability of a sensor to be selected as a $\mathrm{CH}$ versus the D3N and RPS. Also, here we see 3 zones. When the RPS is low and the number of neighbor nodes (D3N) is high, the possibility that a node to be selected as CH is low. When the RPS is middle and the D3N increases, the possibility that a node to be selected as CH is increased. When the RPS is high and the $\mathrm{D} 3 \mathrm{~N}$ is high, the possibility that a node to be selected as $\mathrm{CH}$ is high.

In Fig. 10, we show the relation between probability of a sensor to be selected as a $\mathrm{CH}$ versus the $\mathrm{D} 3 \mathrm{~N}$ and DCC. With the increase of $\mathrm{D} 3 \mathrm{~N}$ and the decrease of DCC, the possibility of a sensor to be selected as $\mathrm{CH}$ is increased. Different from the results in Fig. 8 and Fig. 9, we see that the shape of the surface is changed proportionally with the changes of parameters. This shows that the RPS and 3DN parameters are more important than $\mathrm{DCC}$ for $\mathrm{CH}$ selection.

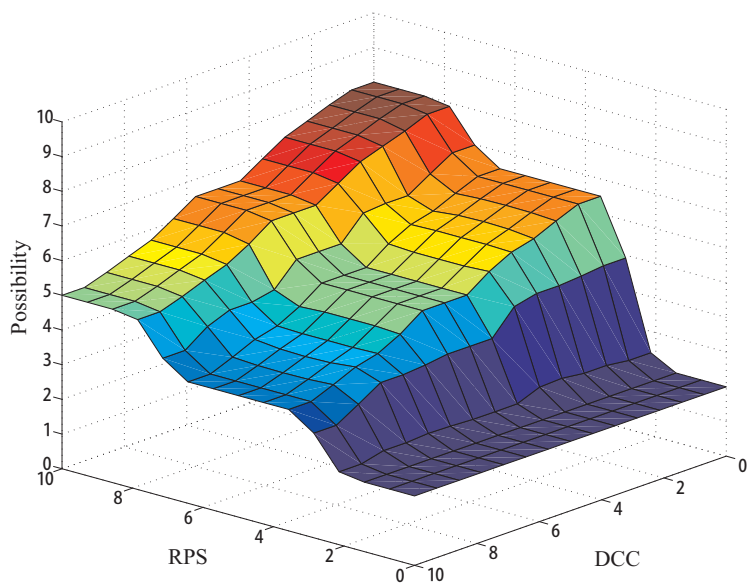

Figure 8. F3N system results (case 1).

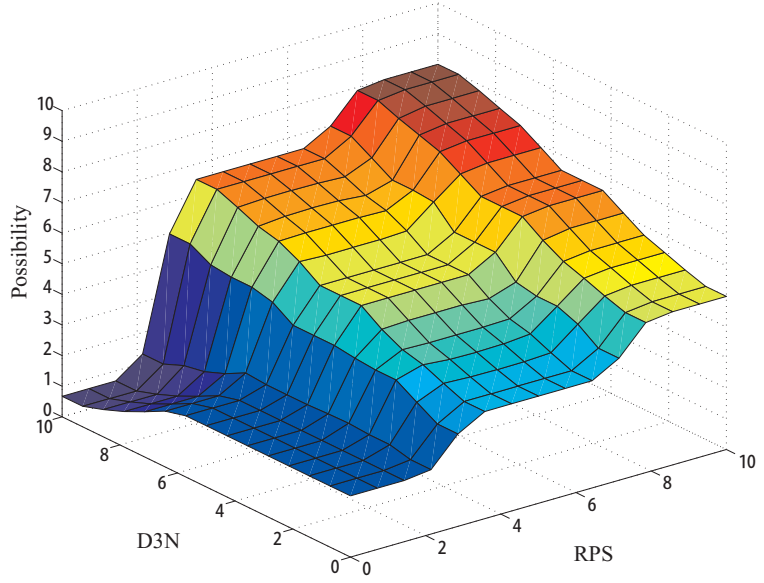

Figure 9. F3N system results (case 2).

\section{Conclusions}

The power conservation in WSN is a very important issue. Conserving power prolongs the lifetime of a node and also the lifetime of the whole network. Clustering is one of the energy-efficient techniques for extending the lifetime of a sensor network. Clustering techniques organize the nodes into clusters where some nodes work as $\mathrm{CH}$ and collect the data from other nodes in the clusters. However, $\mathrm{CH}$ selection is very difficult when many parameters are used for making the decision.

In our previous work, in order to select the $\mathrm{CH}$, we proposed a power reduction algorithm for sensor networks based on fuzzy logic and number of neighbor nodes. In this paper, we implement a simulation system for clustering algorithms in sensor networks. 


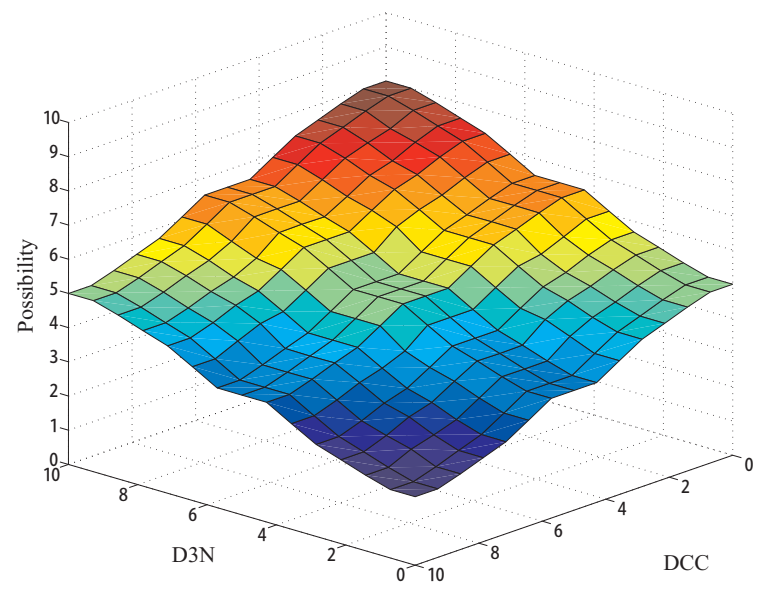

Figure 10. F3N system results (case 3).

We evaluate LEACH and F3N by some simulation results. Presently, we have implemented LEACH algorithm in NS-2. However, F3N is implemented in MATLAB. We are working to implement also F3N system in NS-2 in order to compare their performance.

\section{ACKNOWLEDGMENT}

This work is support by a Grant-in-Aid for scientific research of Japanese Society for the Promotion of Science (JSPS). The authors would like to thank JSPS for the financial support.

\section{REFERENCES}

[1] I. F. Akyildiz, W. Su, Y. Sankarasubramaniam, and E. Cayirci, "Wireless sensor networks: a survey," Computer Networks, vol. 38, no. 4, pp. 393-442, 2002.

[2] I. F. Akyildiz and I. H. Kasimoglu, "Wireless sensor and actor networks: research challenges," Ad Hoc Networks, vol. 2, no. 4, pp. 351-367, 2004.

[3] S. Giordano and C. Rosenberg, "Topics in ad hoc and sensor networks," IEEE Communication Magazine, vol. 44, no. 4, pp. 97-97, 2006.

[4] J. N. Al-Karaki and A. E. Kamal, "Routing techniques in wireless sensor networks: a survey," IEEE Wireless Communication, vol. 11, no. 6, pp. 6-28, December 2004.

[5] M. Chatterjee, S. K. Das, and D. Turgut, "Wca: a weighted clustering algorithm for mobile ad hoc networks," Journal of Cluster Computing, vol. 5, no. 2, pp. 193-204, April 2002.

[6] S. Banerjee and S. Khuller, "A clustering scheme for hierarchical control in multi-hop wireless networks," Proc. of IEEE INFOCOM-2001, pp. 1028-1037, 2001.
[7] W. P. Chen, J. C. How, and L. Sha, "Dynamic clustering for acoustic target tracking in wireless sensor networks," IEEE Trans. on Mobile Computing, vol. 3, no. 3, pp. 258-271, JulySeptember 2004.

[8] S. Basagni, "Distributed clustering for ad hoc networks," International Symposium of Parallel Architectures, Algorithms and Networks (I-SPAN'99), pp. 310-315, 1999.

[9] A. D. Amis, R. Prakash, T. H. P. Vuong, and D. T. Huynh, "Max-min d-cluster formation in wireless ad hoc networks," Proc. of IEEE INFOCOM-2000, pp. 32-41, 2000.

[10] H. Chan and A. Perrig, "Ace: An emergent algorithm for highly uniform cluster formation," Proc. of European Workshop on Wireless Sensor Networks (EWSN-2004), pp. 154171, 2004.

[11] W. B. Heinzelman, A. P. Chandrakasan, and H. Balakrishnan, "An application-specific protocol architecture for wireless microsensor networks," IEEE Transactions on Wireless Communications, vol. 1, no. 4, pp. 660-670, October 2004.

[12] W. R. Heinzelman, A. Chandrakasan, and H. Balakrishnan, "Energy-efficient communication protocol for wireless microsensor networks," Proc. of the 33rd Annual Hawaii International Conference on System Sciences (HICSS), pp. 3005-3014, 2000.

[13] S. Lindsey, C. Raghavendra, and K. M. Sivalingam, "Data gathering algorithms in sensor networks using energy metrics," IEEE Trans. on Parallel and Distributed Systems, vol. 13, no. 9, pp. 924-935, 2002.

[14] P. M. L. Chan, R. E. Sheriff, Y. Hu, P. Conforto, and C. Tocci, "Mobility management incorporating fuzzy logic for a heterogeneous ip environment," IEEE Communications Magazine, vol. 39, no. 12, pp. 42-51, 2001.

[15] L. Barolli, A. Koyama, T. Suganuma, and N. Shiratori, "Gaman: a ga based qos routing method for mobile adhoc networks," Journal of Interconnection Networks (JOIN), vol. 4, no. 3, pp. 251-270, 2003.

[16] J. Anno, L. Barolli, F. Xhafa, and A. Durresi, "A cluster head selection method for wireless sensor networks based on fuzzy logic," Proc. of IEEE TENCON-2007, pp. CD-ROM, 4 pages, 2007.

[17] Q. Liang, "A design methodology for wireless personal area networks with power efficiency," Proc. of the Wireless Communications and Networking (WCNC), vol. 3, pp. 1475-1480, 2003.

[18] J. Anno, L. Barolli, A. Durresi, F. Xhafa, and A. Koyama, "A cluster head decision system for sensor networks using fuzzy logic and number of neighbor nodes," Proc. of IEEE Ubi-media 2008, pp. 50-56, 2008.

[19] J. Anno, L.Barolli, F. Xhafa, A. Durresi, and A. Koyama, "Performance evaluation of two-fuzzy based cluster head selection systems for wireless sensor networks," Journal of Mobile Information Systems (MIS), vol. 4, no. 4, pp. 297312, 2008.

[20] J. M. Mendel, "Fuzzy logic systems for engineering: a tutorial," Proc. of the IEEE, vol. 83, no. 3, pp. 345-377, 1995. 